\title{
Effect of SNR of Input Signal on the Accuracy of a Ratiometric Wavelength Measurement System
}

\author{
Ginu Rajan \\ Technological University Dublin, ginu.rajan@tudublin.ie \\ Qian Wang \\ Technological University Dublin, qian.wang@tudublin.ie \\ Gerald Farrell \\ Technological University Dublin, gerald.farrell@tudublin.ie
}

See next page for additional authors

Follow this and additional works at: https://arrow.tudublin.ie/engscheceart

Part of the Engineering Commons

\section{Recommended Citation}

Rajan, G., Wang, Q., Farrell, G., Semenova, Y. and Wang, P.: "Effect of SNR of an input signal on the accuracy of a ratiometric wavelength measurement system”, , Vol. 49, Issue 5, pp. 1022-1024. May 2007. doi;10.1002/mop.22328.

This Article is brought to you for free and open access by the School of Electrical and Electronic Engineering at ARROW@TU Dublin. It has been accepted for inclusion in Articles by an authorized administrator of ARROW@TU Dublin. For more information, please contact arrow.admin@tudublin.ie, aisling.coyne@tudublin.ie, gerard.connolly@tudublin.ie.

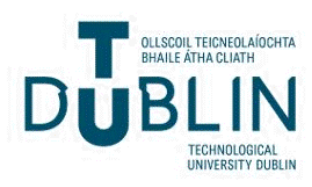




\section{Authors}

Ginu Rajan, Qian Wang, Gerald Farrell, Yuliya Semenova, and Pengfei Wang

This article is available at ARROW@TU Dublin: https://arrow.tudublin.ie/engscheceart/96 


\title{
Effect of SNR of input signal on the accuracy of a ratiometric wavelength measurement system
}

\author{
Ginu Rajan, Qian Wang, Gerald Farrell, Yuliya Semenova and Pengfei Wang \\ Applied Optoelectronics Centre, School of Electronics and Communication, \\ Dublin Institute of Technology, Kevin Street, Dublin-8, Ireland \\ ginu.rajan@student.dit.ie
}

\begin{abstract}
:
The impact of a change in SNR of the input signal on the accuracy of a ratiometric wavelength measurement system is studied. The variation of the output ratio of the system due to the change in SNR of the input signal is modeled and the experimental investigation has been carried out, and both indicate that the accuracy is influenced significantly by variations in the SNR of the input signal. The demonstration example shows a wavelength shift of $0.055 \mathrm{~nm}$ when the SNR of the input signal varies from $47 \mathrm{~dB}$ to $42 \mathrm{~dB}$ (the measurable range is from $1500 \mathrm{~nm}$ to $1600 \mathrm{~nm}$ and the edge filter used has a slope of $0.15 \mathrm{~dB} / \mathrm{nm})$.
\end{abstract}

Keywords:- Ratiometric system, edge filter, SNR 


\section{Introduction}

Wavelength measurement is involved in many optical systems such as dense wavelength division multiplexing (DWDM), and optical sensing based on fiber Bragg gratings (FBGs) [1,2] where an accurate measurement of wavelength is needed. Among different wavelength measurement schemes, a ratiometric detection scheme [3-6] employing an edge filter has a simple configuration which uses the transition region of the filter's transmission response and converts wavelength measurement to a signal intensity measurement. The different edge filters used are bulk thin film filters [3], biconical fiber filters [4], fiber gratings [5], macro bend singlemode fiber [6] etc. In principle a ratiometric scheme should allow for the measurement of the wavelength of an input signal without a dependency on other parameters of the input signal such as signal power or SNR. In practice, the input signal has a limited signal-to-noise ratio (SNR), and it has been proven in a previous investigation [7] that the SNR limits the slope of the edge filter and measurement range of the system. In this letter the accuracy of a ratiometric system is investigated theoretically and experimentally, taking into account the effect of the SNR of the input signal, which indicates that variations in the SNR have a significant influence on the system's accuracy.

\section{Analysis of the impact of SNR on ratiometric system}

The schematic configuration of the ratiometric wavelength measurement system based on a macrobending fiber filter is shown in Fig.1. The input signal is split into two signals. One passes through a reference arm and the other passes through the bending fiber. The macrobending fiber acts as an edge filter as in Ref. [6]. Two photodetectors are placed at the ends of both arms. The system effectively operates as a discriminator, where the ratio of the power levels reaching the photodetectors is wavelength dependent. The wavelength of the input signal can be determined, 
assuming a suitable calibration has taken place, using the ratio of the electrical outputs of the two photodetectors [7], which is:

$$
R\left(\lambda_{0}\right)=-10 \log _{10}\left[\frac{\int S_{1 \lambda_{0}}(\lambda) \cdot I_{\lambda_{0}}(\lambda) \cdot T_{f}(\lambda) d \lambda}{\int S_{2 \lambda_{0}}(\lambda) I_{\lambda_{0}}(\lambda) d \lambda}\right]
$$

Where $S_{1}(\lambda)$ and $S_{2}(\lambda)$ are the transmission responses of the arms of the splitter. $T_{f}(\lambda)$ is the transmission response of the edge filter and $I_{\lambda_{0}}(\lambda)$ is the spectrum of the input signal. In practice, the light from a tunable laser or reflected by an FBG element has a limited signal-to-noise ratio. Assuming for convenience a source with a power of 0 $\mathrm{dBm}$ at the peak wavelength, then as in Ref. [7], the spectrum of such an input signal can be modelled as:

$10 \log _{10}\left[I_{\lambda_{0}}(\lambda)\right]=\left\{\begin{array}{cc}10 \log _{10}\left[\exp \left(-4 \ln 2 \frac{\left(\lambda-\lambda_{0}\right)^{2}}{\Delta \lambda 0^{2}}\right],\right. & |\lambda-\lambda 0| \leq \Omega \\ -S(\lambda)+\text { Rand.Rs, } & |\lambda-\lambda 0|>\Omega\end{array}\right.$

$S$ is the SNR for the source in $\mathrm{dB}$. To describe the random fluctuations in the noise floor of the optical source, the term Rand.Rs is used, where Rand is a random number (between +1 and -1 ) and $R s$ is a parameter in $\mathrm{dB}$ which dictates the peak fluctuation in the SNR and is dependent on the nature of the source. $\Omega$ is a parameter which is determined by the noise level and can be determined for a source with a given SNR from the relationship $10 \log _{10}\left[\exp \left(-4 \ln 2 \frac{\Omega^{2}}{\Delta \lambda_{0}^{2}}\right)\right]=-S$.

Using the above equations a system with an edge filter with a discrimination range of $25 \mathrm{~dB}$ and a $3 \mathrm{~dB}$ coupler which splits the input signal is modelled for different SNR values, for a wavelength range $1500 \mathrm{~nm}$ to $1600 \mathrm{~nm}$. The modelled results are shown in Fig. 2 and it can be seen that different SNRs produce a discrimination characteristic which is not the linear ideal. In practice, when the SNR of the input signal changes, 
the measured wavelength will be an error where the original calibration curve designed for a different SNR is used. Fig. 3 shows a numerical example of the influence of SNR on output ratio for a fixed wavelength. Assume the wavelength of the input signal is $1520 \mathrm{~nm}$ and the slope of the edge filter is $0.25 \mathrm{~dB} / \mathrm{nm}$. When the SNR decreases from $50 \mathrm{~dB}$ to $45 \mathrm{~dB}$, the calculated ratio variation is $0.0042 \mathrm{~dB}$ which would leads to a wavelength error of $0.017 \mathrm{~nm}$. Thus it is seen that for a system SNR changes will have a significant impact on the wavelength accuracy of the system.

For low SNR values another source of error can also be deduced from Fig. 2. For high values of SNR, for example $50 \mathrm{~dB}$, the slope of the characteristic is close to the ideal. However for low values of SNR, below $30 \mathrm{~dB}$, the slope is much lower. For example over the wavelength range $1550 \mathrm{~nm}$ to $1600 \mathrm{~nm}$ the slope with a $50 \mathrm{~dB}$ SNR is 0.25 $\mathrm{dB} / \mathrm{nm}$, whereas for an SNR of $30 \mathrm{~dB}$ the slope is less than $0.1 \mathrm{~dB} / \mathrm{nm}$. The consequence of the reduced slope is decreased measurement accuracy, particularly for low optical input powers, because of electrical noise and the limited resolution of the electrical subsystems that process the photodiode electrical power outputs.

\section{Experimental Investigation}

To verify the influence of SNR on the ratiometric system accuracy experimentally, a ratiometric wavelength measurement system based on the macrobending singlemode fiber was built with a slope of approximately $0.15 \mathrm{~dB} / \mathrm{nm}$. The test source used was a tunable laser for which the SNR varies from $47 \mathrm{~dB}$ to $42 \mathrm{~dB}$ when tuning the power from $0 \mathrm{dBm}$ to $-5 \mathrm{dBm}$ respectively. The system is calibrated for the discriminator characteristic for an SNR of $47 \mathrm{~dB}$. The ratio is measured for a wavelength range of $1500 \mathrm{~nm}$ to $1600 \mathrm{~nm}$ at $0 \mathrm{dBm}$ and $-5 \mathrm{dBm}$, respectively. The variation in the ratio is presented in Fig. 4. A comparison with the simulated results is also presented which show that both are in good agreement and which clearly emphasises the effect of SNR 
on the system. To estimate the wavelength inaccuracy with the changes in the SNR, the variation in ratio and the corresponding wavelength shift for $1520 \mathrm{~nm}$ is obtained. The measured ratio variation at different SNR and its comparison with the simulated results are shown in Fig. 5. The measured and simulation results show how the ratio of the system changes with SNR. It is seen that the variation of the ratio is $0.0087 \mathrm{~dB}$ when the SNR decreases from $47 \mathrm{~dB}$ to $42 \mathrm{~dB}$. Any change in ratio leads to a wavelength measurement error. To illustrate this, the corresponding variation in wavelength measured is shown in Fig. 6 for a step change in SNR. It can be seen that the wavelength error reaches $0.055 \mathrm{~nm}$ due to the change of SNR of $5 \mathrm{~dB}$.

\section{Conclusion}

The wavelength inaccuracy in a ratiometric measurement system employing an edge filter has been studied theoretically and experimentally. It has been shown that a different SNR for the input signal corresponds to different effective discrimination characteristics. It is been demonstrated that for a wavelength of $1520 \mathrm{~nm}$ when the SNR varies from $47 \mathrm{~dB}$ to $42 \mathrm{~dB}$ the measured wavelength error is $0.055 \mathrm{~nm}$ for a system with a slope of $0.15 \mathrm{~dB} / \mathrm{nm}$. This suggests that when there is a change in the SNR of the input signal, e.g., where a ratiometric system is switched to a different source, a re-calibration will be required due to the influence of the SNR on the system's accuracy. 


\section{References}

1. B. Mason, S. P. DenBaars and L. A. Coldren, Tunable sampled-grating DBR lasers with integrated wavelength monitors, IEEE Photon. Technol. Lett. 10 (1998), 1085-1087.

2. M.A Davis and A.D Kersey, All fiber Bragg grating sensor demodulation technique using a wavelength division coupler, Electron. Lett. 30 (1994), 75-76.

3. S.M Melle, K. Liu and R. M. Measures, A passive wavelength demodulation system for guided wave Bragg grating sensors, IEEE Photonics Technol. Lett. 4 (1992), 516-518.

4. A.B.L Ribeiro, L.A Ferreira, M. Tsvekov and J.L Santos, All-fiber interrogation technique for fiber Bragg sensors using biconical fiber filter, Electron. Lett. 32 (1996), 382-383.

5. Y. Liu, L. Zhang and I Bennion, Fabricating fibre edge filters with arbitrary spectral response based on tilted chirped grating structures, Meas.Sci. Technol. 10 (1999), L1-L3.

6. Qian Wang, Gerald Farrell, Thomas Freir, Ginu Rajan, Pengfei Wang, Low-cost wavelength measurement based on a macrobending single-mode fiber, Opt. Lett. 31 (2006), 1785-1787.

7. Qian Wang, Gerald Farrell, Thomas Freir, Study of transmission response of edge filters employed in wavelength measurements, Appl. Opt. 44 (2005), 77897792. 


\section{Figure captions:}

Figure. 1. Schematic of a fiber bend loss ratiometric wavelength measurement system

Figure. 2. Ratio of an edge filter at different SNR

Figure. 3. Variation in ratio when SNR changes from $50 \mathrm{~dB}$ to $45 \mathrm{~dB}$

Figure. 4. Ratio variation when SNR changes from $47 \mathrm{~dB}$ to $42 \mathrm{~dB}$ and its comparison with simulation result

Figure. 5. Comparison of measured and modelled ratio variation

Figure. 6. The measured wavelength shift when SNR changes from $47 \mathrm{~dB}$ to $42 \mathrm{~dB}$ 
Figure 1

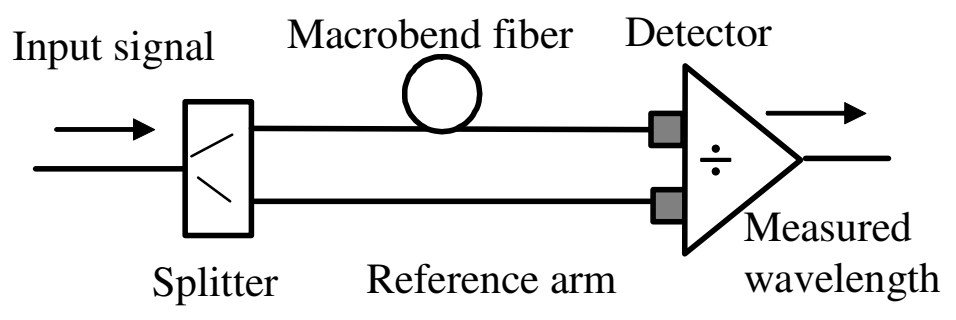


Figure 2

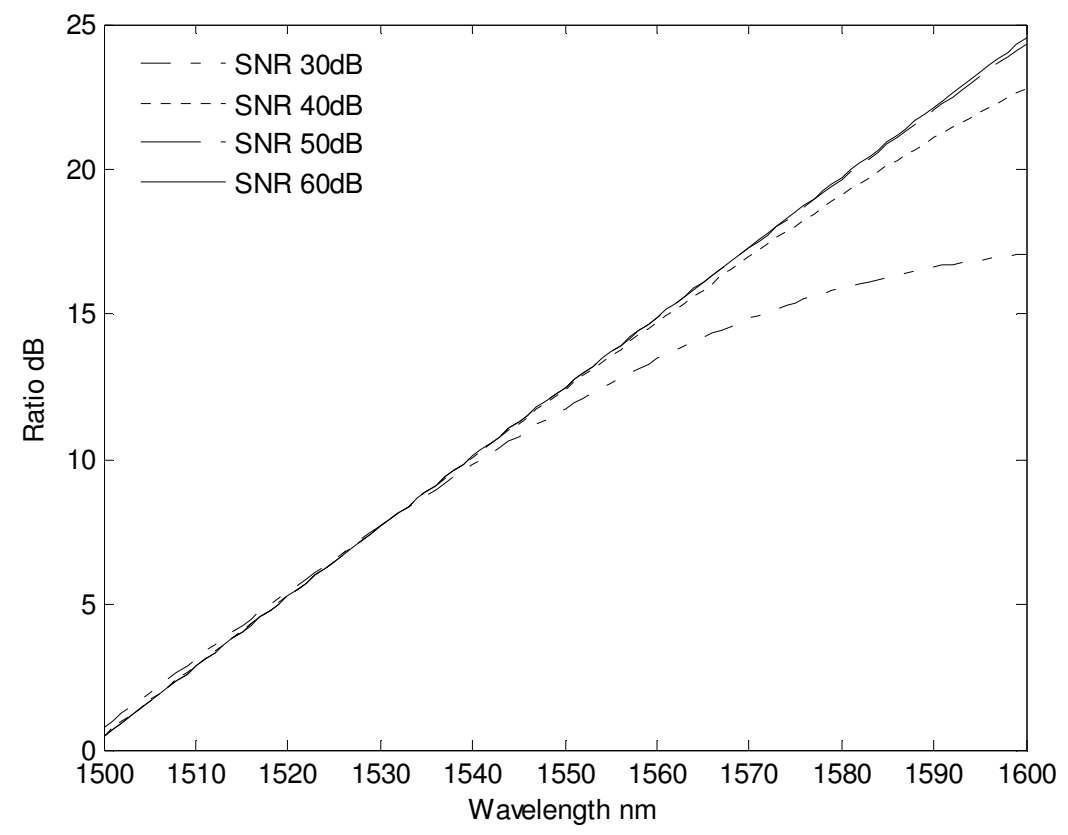


Figure 3

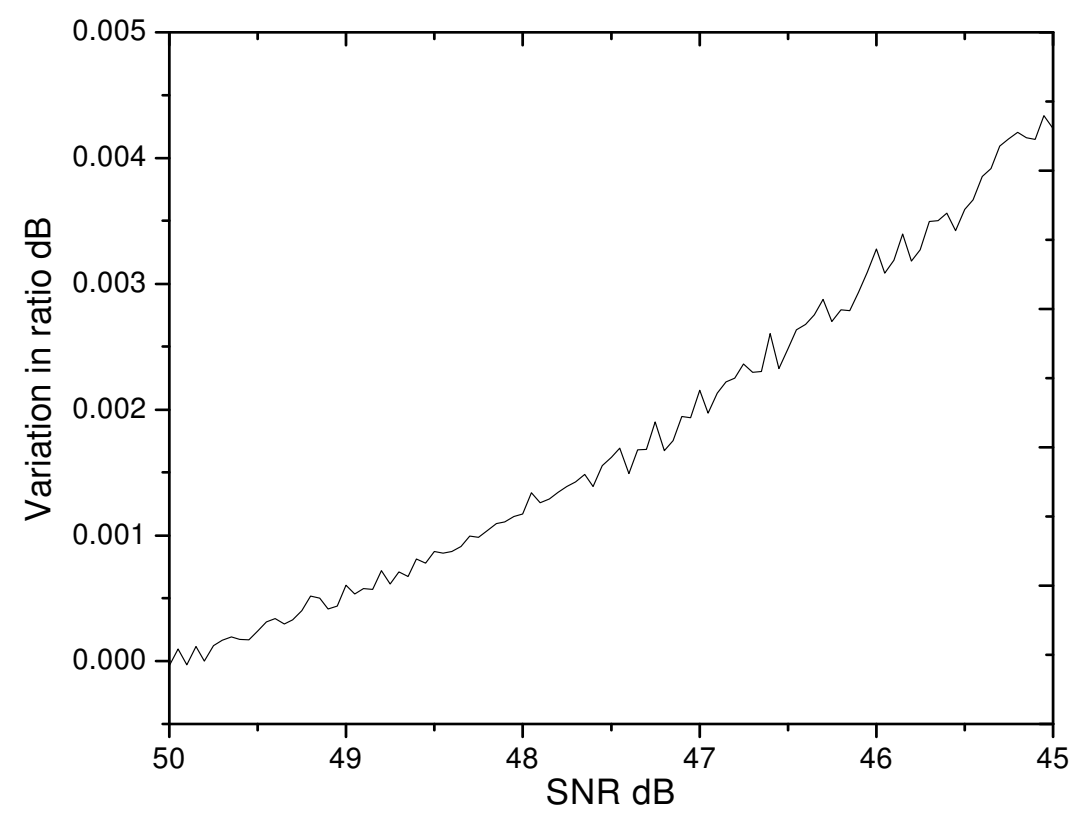


Figure 4

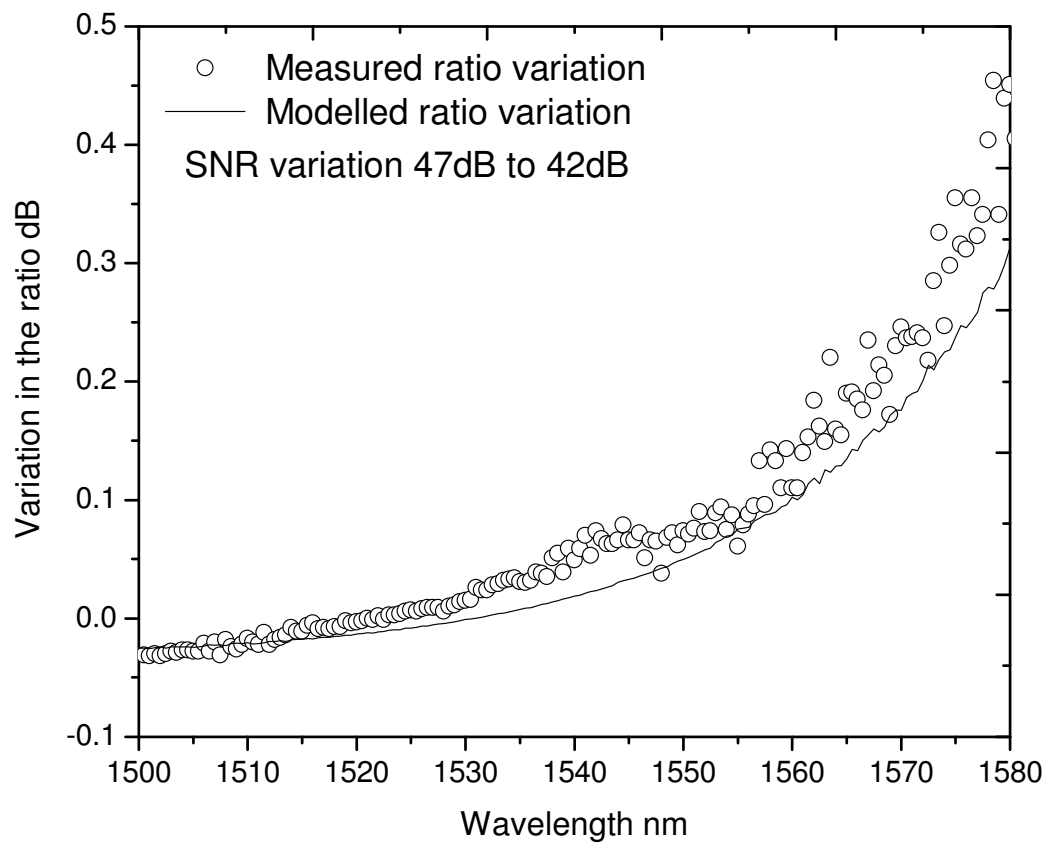


Figure 5

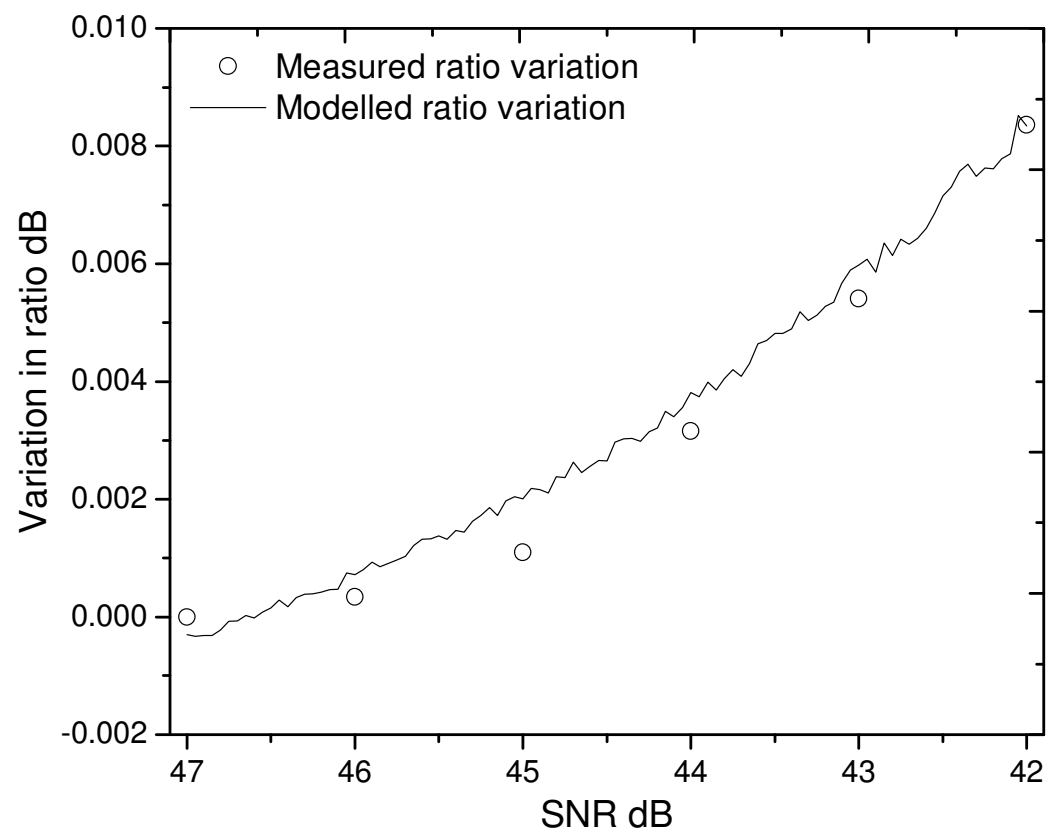


Figure 6

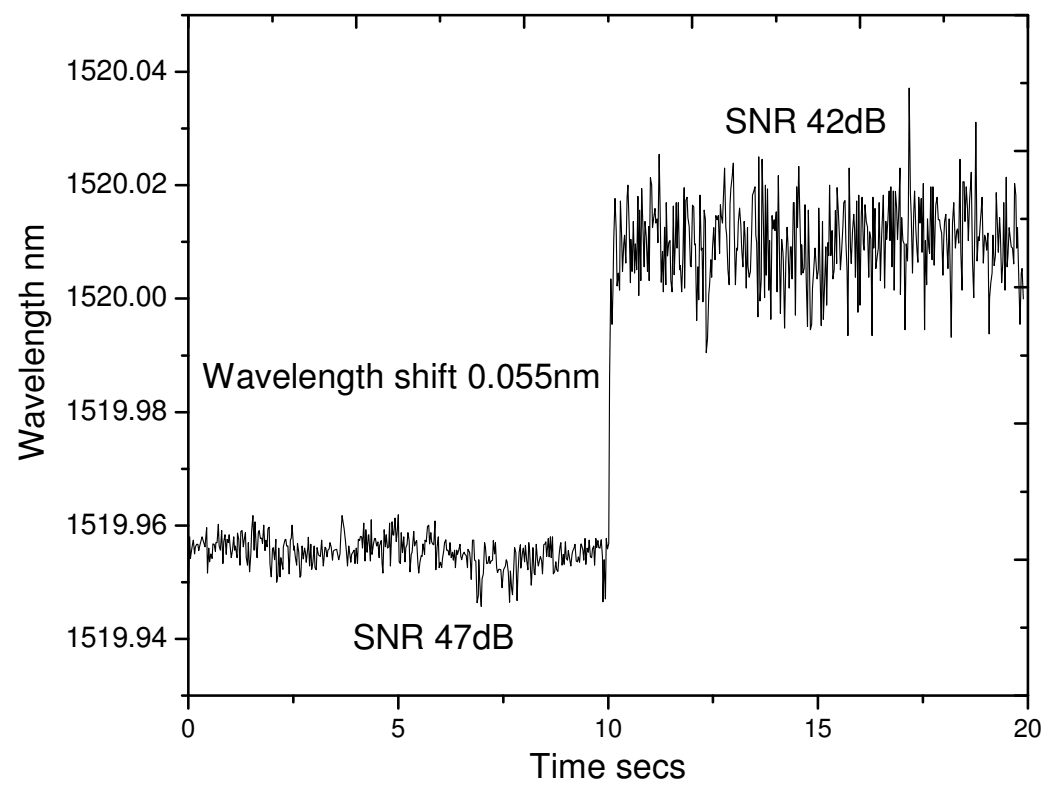

